\title{
Proportion of clinically significant prostate cancer diagnosed by systematic template biopsy after negative pre-biopsy multiparametric magnetic resonance imaging and predictive value of prostate-specific antigen density
}

Mohammad Haroon; Paul Sathiadoss; Rodney H. Breau; Ilias Cagiannos; Trevor Flood; Luke T. Lavallee; Christopher Morash; Nicola Schieda The Ottawa Hospital, Ottawa, ON, Canada

Cite as: Haroon M, Sathiadoss P, Breau RH, et al. Proportion of clinically significant prostate cancer diagnosed by systematic template biopsy after negative pre-biopsy multiparametric magnetic resonance imaging and predictive value of prostate-specific antigen density. Can Urol Assoc J 2021 October 18; Epub ahead of print.

http://dx.doi.org/10.5489/cuaj.7455

Published online October 18, 2021

Corresponding author: Dr. Nicola Schieda, The Ottawa Hospital, Ottawa, ON, Canada; nschieda@toh.on.ca

$* * *$

\section{Introduction}

Biopsy deferral in men with a negative prostate multiparametric magnetic resonance imaging (mpMRI) is gaining popularity in clinical practice, as mpMRI becomes integrated into prostate cancer (PCa) diagnostic pathways [1]. However, clinically significant (cs)PCa may be diagnosed at subsequent template biopsy in men with negative pre-biopsy mpMRI [2]. This study evaluated the proportion of men with csPCa diagnosis at template biopsy following a negative mpMRI and evaluated PSA density as a predictor of future positive template biopsy.

\section{Methods}

This IRB-approved, retrospective, single center, cross-sectional study identified XX consecutive men with negative mpMRI (PI-RADS score 1 or 2) performed before TRUSguided systematic template biopsy between January 12013 and August 1 2021. mpMRI technique (Table 1) and reporting was compliant with PI-RADS [3]. Standard 12-sample TRUS-guided template biopsies were performed using 18-gauge side-cutting needles. Age, clinical indication, PSA, prostate volume (measured on MRI using ellipsoid volume calculation), PSAD (PSA/volume) and clinical stage were recorded. The proportion of men with CS-PCa (defined as International Society of Urogenital Pathology [ISUP] grade group $\geq 2 \mathrm{PCa}$ ) diagnosed at subsequent template biopsy was recorded. PSAD comparison between 
groups was performed using an independent t-test and empiric receiver operator characteristic (ROC) curves generated.

\section{Results}

A summary of patient clinical variables by indication is provided in Table 2 . Considering any PCa, 40.4\% (42/104) cancers were diagnosed with 58.1\% (25/43) in biopsy-naïve and $27.9 \%(17 / 61)$ in the previous negative template biopsy groups $(\mathrm{p}<0.01)$. Overall, $11.5 \%$ $(12 / 104)$ men had CS-PCa (ISUP $\geq 2$ ) diagnosed at template biopsy; XX20.9\% (9/43) in the biopsy-naïve group and 4.9\% (3/61) in the previous negative template biopsy group $(\mathrm{p}<0.01)$, Table 2 .

There was no difference in PSA or PSAD comparing the biopsy-naïve to the previous negative template biopsy group ( $p>0.05)$, which were combined for all further analyses. Both PSA $(\mathrm{p}<0.01)$ and PSAD $(\mathrm{p}<0.01)$ were higher in men with CS-PCa diagnosed at subsequent template biopsy, but otherwise did not differ by indication $(\mathrm{p}=0.62)$ or diagnosis of any $\mathrm{PCa}(\mathrm{p}>0.05)$. Area under the ROC curve for diagnosis of CS-PCa in the pre-biopsy group using PSA and PSAD were: 0.59 (95\% CI 0.0.42, 0.76) and $0.69(0.0 .53,0.85)$, Figure 1. The optimal cutpoint for PSAD derived by the method of Youden was $\geq 0.18 \mathrm{ng} / \mathrm{mL}$ with sensitivity and specificity of $75 \%$ and $57 \%$ respectively. Patients were stratified into 3 groups by $P S A D \geq 0.10, \geq 0.15$ and $\geq .0 .20 \mathrm{ng} / \mathrm{mL}$, as suggested previously [4], and diagnostic accuracy calculated at each threshold (Table 3 ).

\section{Discussion}

In this study, PI-RADS score 1 or 2 plus PSAD was useful to predict eventual CS-PCa diagnosis in biopsy naïve men and those with previous negative template biopsy who underwent subsequent template biopsy. The optimal cutpoint of PSAD $\geq 0.18$ yielded highest accuracy for diagnosis of CS-PCa at subsequent template biopsy.

Data regarding biopsy deferral in at risk men without a prior PCa diagnosis (including biopsy naïve men and those with previous negative biopsy) informed by negative mpMRI and PSAD are favorable. Previous studies have shown an eventual clinically significant $\mathrm{PCa}$ diagnosis rate in this population ranging between $4.4 \%$ and $17 \%[2,5]$ and have consistently shown that PSAD differs between those men with an eventual CS-PCa diagnosis and those with negative results at subsequent template biopsy. A recent study by Deniffel et al. demonstrated that a PI-RADS plus PSAD $\geq 0.1 \mathrm{ng} / \mathrm{mL}$ strategy could safely reduce unnecessary biopsy without missing CS-PCa outperforming other MRI-based risk models and did not require calibration [6]. To our knowledge, the optimal PSAD level to optimally define a patient with negative pre-biopsy mpMRI who may benefit from template biopsy has not been derived. In a 2020 review that included 3006 biopsy naïve men in 5 studies showed that MRI-negative men with low-risk PSAD $(\leq 0.10 \mathrm{ng} / \mathrm{mL})$ have $3 \%$ risk of CS-PCa, with low-intermediate risk PSAD $(\leq 0.15 \mathrm{ng} / \mathrm{mL})$ have $7 \%$ risk of CS-PCa and 
those with high-risk PSAD $(\geq 0.20 \mathrm{ng} / \mathrm{mL})$ have $18 \%$ risk of CS-PCa [4]. Our data are strikingly similar to this review suggesting that $\mathrm{PSAD}<0.1$, biopsy can be avoided and PSAD $>0.2$, biopsy should be considered.

Our study has limitations. Our cohort consisted of men having pre-biopsy MRI with or without a preceding negative template biopsy and the mixed indications may be considered a limitation. Our sample is relatively small, consisting of approximately 104 men split relatively evenly into a biopsy naïve and previous negative template biopsy cohort. Not all patients with negative MRI undergo template biopsy at our institution and our results could be biased by those men who were referred for template biopsy compared to a consecutive cross-section of men with negative MRI. In conclusion, our results support a PIRADS score 1 or 2 plus PSAD level strategy in biopsy naïve men and those with previous negative template biopsy, which may predict which men may benefit from a template biopsy after negative MRI. The optimal PSAD level which should be used clinically with PI-RADS scoring in this clinical context requires further study.

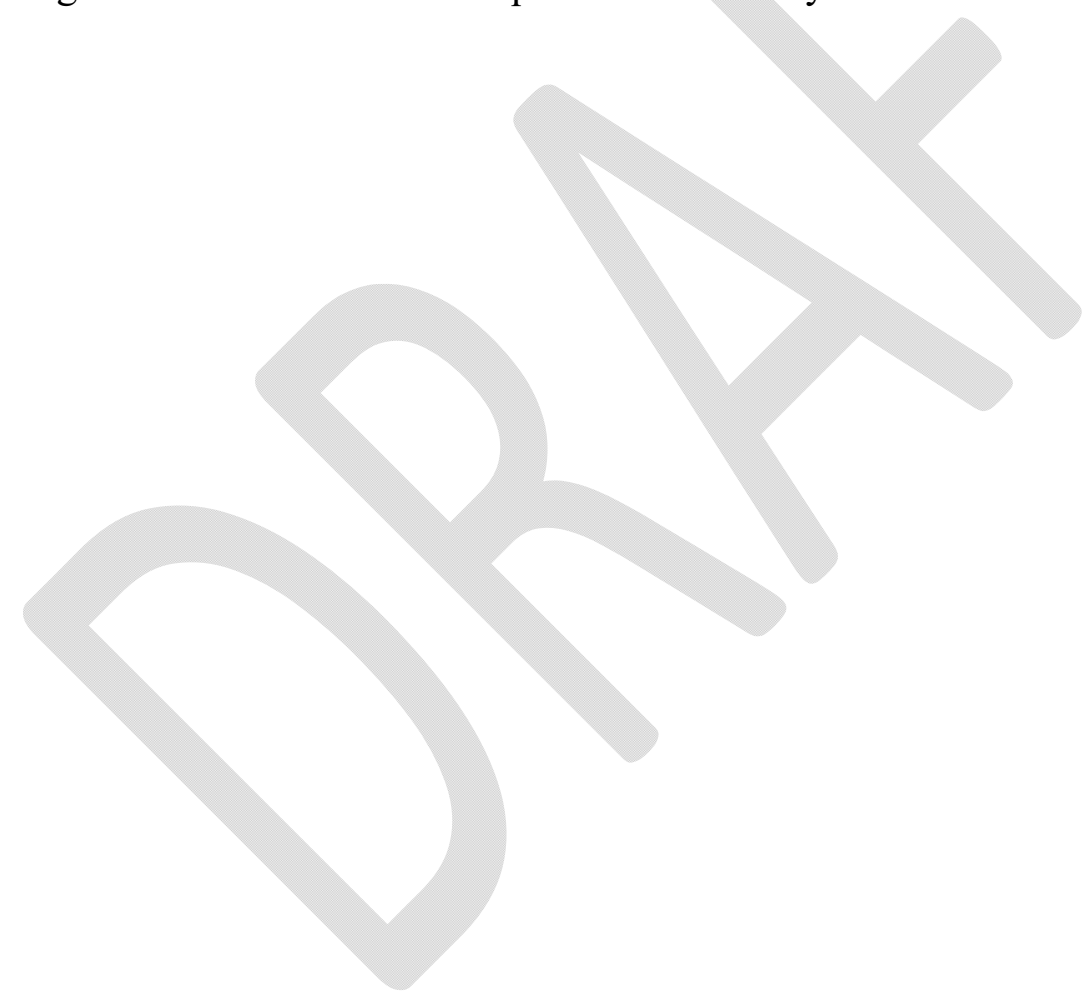




\section{References}

1. Haider MA, B.J., Chin J, Loblaw A, Perlis N, Schieda N., Multiparametric Magnetic Resonance Imaging in the Diagnosis of Clinically Significant Prostate Cancer., in Program in Evidence-Based Care Guideline No.: 27-2 Version 2. 2021, Toronto (ON): Ontario Health (Cancer Care Ontario).

2. Buisset, J., et al., Negative Pre-biopsy Magnetic Resonance Imaging and Risk of Significant Prostate Cancer: Baseline and Long-Term Follow-up Results. Journal of Urology, 2020. 205.

3. Purysko, A., et al., PI-RADS Version 2: A Pictorial Update. Radiographics, 2016. 36: p. 150234.

4. Schoots, I. and A. Padhani, Risk-adapted biopsy decision based on prostate MRI and PSA-density for enhanced biopsy avoidance in first prostate cancer diagnostic work-up. BJU Int, 2020. 127.

5. Norris, J., et al., What Type of Prostate Cancer Is Systematically Overlooked by Multiparametric Magnetic Resonance Imaging? An Analysis from the PROMIS Cohort. Eur Urol, 2020. 78.

6. Deniffel, D., et al., Avoiding Unnecessary Biopsy: MRI-based Risk Models versus a PI-RADS and PSA Density Strategy for Clinically Significant Prostate Cancer. Radiology, 2021: p. 204112. 


\section{Figures and Tables}

Fig. 1. Empiric receiver operator characteristic (ROC) curve for diagnosis of clinically significant prostate cancer by prostate-specific antigen (PSA) density biopsy-naive men and those with previous negative template biopsy.

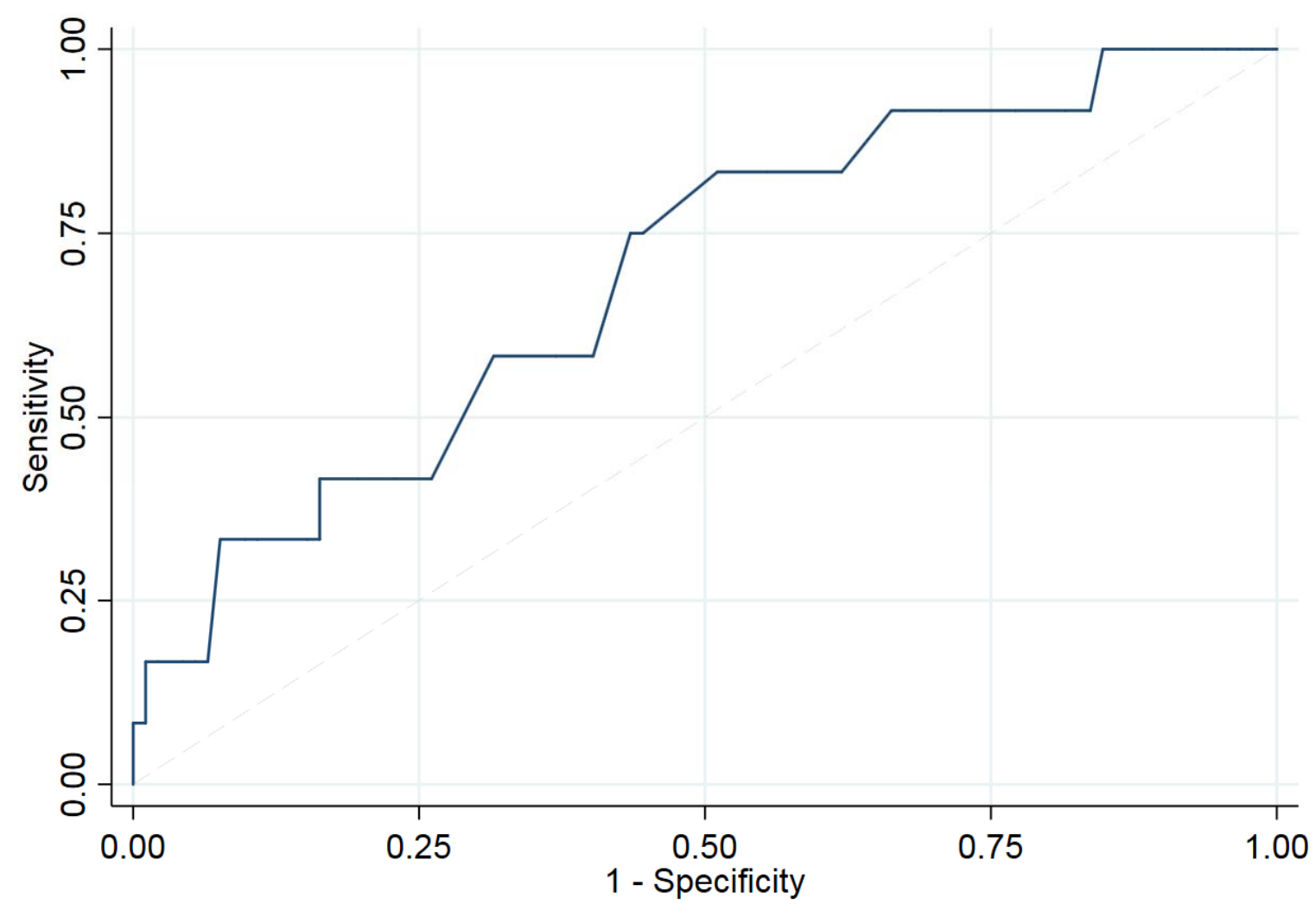

Area under $\mathrm{ROC}$ curve $=0.6925$ 
Table 1. Multiparametric MRI technique ${ }^{a}$ performed during the study period

\begin{tabular}{|c|c|c|c|c|c|c|c|c|c|c|c|}
\hline & $\begin{array}{c}\text { Imaging } \\
\text { plane }\end{array}$ & $\begin{array}{l}\text { Field of } \\
\text { view } \\
(\mathrm{mm})\end{array}$ & $\begin{array}{c}\text { Matrix } \\
\text { size }\end{array}$ & $\begin{array}{c}\text { Slice } \\
\text { thickness/ } \\
\text { gap (mm) }\end{array}$ & $\begin{array}{l}\text { TR/TE } \\
\text { (msec) }\end{array}$ & $\begin{array}{l}\text { Echo } \\
\text { train } \\
\text { length }\end{array}$ & $\begin{array}{c}\text { Flip } \\
\text { angle }\end{array}$ & $\begin{array}{l}\text { Acceleration } \\
\text { factor }\end{array}$ & $\begin{array}{c}\text { Receiver } \\
\text { bandwidth } \\
\text { (Hz/Voxel) }\end{array}$ & $\begin{array}{l}\text { Acquisition } \\
\text { time (min) }\end{array}$ & $\begin{array}{l}\text { Number of } \\
\text { signals } \\
\text { averaged }\end{array}$ \\
\hline $\mathrm{T} 2 \mathrm{TSE}^{\mathrm{b}}$ & $\begin{array}{c}\text { Coronal } \\
\text { Sagittal } \\
\text { Axial }\end{array}$ & $220 \times 220$ & $320 \times 256$ & $\begin{array}{l}4.0 / 0 \\
3.0 / 0 \\
3.0 / 0\end{array}$ & $\begin{array}{c}3890- \\
5250 / \\
105-125\end{array}$ & $27-35$ & 111 & N/A & 122 & $\begin{array}{l}4 \min \\
4 \min \\
4 \min \end{array}$ & $1-2$ \\
\hline $\mathrm{DWI}^{\mathrm{c}}$ & Axial & $220 \times 220$ & $128 \times 80$ & $4.0 / 0$ & $4200 / 90$ & 1 & 90 & 2 & 1950 & $5 \mathrm{~min}$ & $4-10$ \\
\hline $\begin{array}{l}\text { T1 GRE } \\
\text { dynamic } \\
\text { contrast }\end{array}$ & Axial & $220 \times 220$ & $128 \times 128$ & $4.0 / 0$ & $4.3 / 1.3$ & $\mathrm{~N} / \mathrm{A}$ & 12 & 2 & 488 & $2 \min$ & 1 \\
\hline
\end{tabular}


channels) with activated spine coils (12 channels). Clinical 3 Tesla systems: TRIO Tim (Siemens Healthcare) and Discovery $750 \mathrm{~W}$ (General Electric, Milwaukee WI). ${ }^{\mathrm{b}}$ Turbo/Fast Spin Echo. ${ }^{\mathrm{c}} \mathrm{DWI}=$ Diffusion weighted imaging performed with spectral fat suppression echo planar imaging with tridirectional motion probing gradients and B values of $0,500,1000 \mathrm{~mm}^{2} / \mathrm{sec}$ with automatic apparent diffusion coefficient map generation derived. b $1500 \mathrm{~mm}^{2} / \mathrm{sec}$ DWI acquired or calculated separately. ${ }^{\mathrm{d} D y n a m i c}$ fast spoiled 3D Gradient Recalled Echo performed with a temporal resolution of 9 seconds after injection of $0.1 \mathrm{mmol} / \mathrm{kg}$ of gadobutrol (Gadovist, Bayer Inc. Toronto, ON) at a rate of $3 \mathrm{~mL} / \mathrm{sec}$. 


\begin{tabular}{|c|c|c|c|}
\hline & \multicolumn{2}{|c|}{$\begin{array}{c}\text { Pre-biopsy } \\
(\mathrm{N}=104)\end{array}$} & $\mathbf{p}$ \\
\hline & $\begin{array}{c}\text { Biopsy-naive } \\
\quad(n=43)\end{array}$ & $\begin{array}{l}\text { Previous negative } \\
\text { template biopsy } \\
(n=61)\end{array}$ & \\
\hline Age (years) & $64 \pm 8$ & $63 \pm 6$ & 0.54 \\
\hline Prostate volume (mL) & $53.5 \pm 23.7$ & $75.6 \pm 42.8$ & $<0.01$ \\
\hline PSA (ng/mL) & $9.2 \pm 6.1$ & $12.7 \pm 10.1$ & 0.11 \\
\hline PSAD (ng/mL) & $0.21 \pm 0.16$ & $0.20 \pm 0.15$ & 0.86 \\
\hline $\begin{array}{l}\text { Diagnosis at subsequent template biopsy } \\
\text { Benign } \\
\text { ISUP }^{3} 1 \\
\text { ISUP } 2 \\
\text { ISUP } 3 \\
\text { ISUP } 4 \\
\text { ISUP } 5\end{array}$ & $\begin{array}{c}41.8 \%(18 / 43) \\
37.2 \%(16 / 43) \\
18.6 \%(8 / 43) \\
0 \%(0 / 43) \\
2.3 \%(1 / 43) \\
0 \%(0 / 43)\end{array}$ & $\begin{array}{c}72.1 \%(/ 61) \\
22.9 \%(14 / 61) \\
1.6 \%(1 / 61) \\
3.2 \%(2 / 61) \\
0 \%(0 / 61) \\
0 \%(0 / 61)\end{array}$ & $<0.01$ \\
\hline
\end{tabular}

ISUP: International Society of Urogenital Pathology; PSA: prostate-specific antigen; PSAD: prostatespecific antigen density.

\begin{tabular}{|c|c|c|c|c|c|}
\hline $\begin{array}{l}\text { PSAD } \\
(\mathrm{ng} / \mathrm{mL})\end{array}$ & $\begin{array}{c}\text { Sensitivity } \\
(95 \% \text { CI) }\end{array}$ & $\begin{array}{l}\text { Specificity } \\
(95 \% \text { CI) }\end{array}$ & $\begin{array}{c}\text { PPV } \\
(95 \% \text { CI })\end{array}$ & $\begin{array}{c}\text { NPV } \\
(95 \% \text { CI })\end{array}$ & $\begin{array}{l}\text { Proportion of } \\
\text { men with } \\
\operatorname{csPCa}^{5}\end{array}$ \\
\hline 0.10 & $\begin{array}{c}92 \% \\
(62-100 \%)\end{array}$ & $\begin{array}{c}19 \% \\
(11-28 \%)\end{array}$ & $\begin{array}{c}13 \% \\
(7-22 \%)\end{array}$ & $\begin{array}{c}94 \% \\
(73-100 \%)\end{array}$ & $\begin{array}{c}1 / 12 \text { csPCa } \\
\text { missed }\end{array}$ \\
\hline 0.15 & $\begin{array}{c}83 \% \\
(52-98 \%)\end{array}$ & $\begin{array}{c}45 \% \\
(34-55 \%)\end{array}$ & $\begin{array}{c}16 \% \\
(8-28 \%)\end{array}$ & $\begin{array}{c}95 \% \\
(84-99 \%)\end{array}$ & $\begin{array}{c}2 / 12 \text { csPCa } \\
\text { missed }\end{array}$ \\
\hline 0.20 & $\begin{array}{c}58 \% \\
(28-85 \%)\end{array}$ & $\begin{array}{c}63 \% \\
(52-73 \%)\end{array}$ & $\begin{array}{c}17 \% \\
(7-32 \%)\end{array}$ & $\begin{array}{c}92 \% \\
(82-97 \%)\end{array}$ & $\begin{array}{c}5 / 12 \mathrm{csPCa} \\
\text { missed }\end{array}$ \\
\hline
\end{tabular}

${ }^{1} \mathrm{n}=104$, including biopsy-naive $(\mathrm{n}=44)$ and men with prior negative template biopsy $(\mathrm{n}=61)$. CI: confidence interval; csPC: clinically significant prostate cancer; ISUP: International Society of Urogenital Pathology; NPV: negative predictive value; PPV: positive predictive value. 